\title{
Minimal Variance Hedging of Options with Student-t Underlying
}

\author{
Klaus Pinn \\ Institut für Theoretische Physik I \\ Universität Münster \\ Wilhelm-Klemm-Str. 9 \\ D-48149 Münster, Germany \\ e-mail: pinn@uni-muenster.de
}

\begin{abstract}
I explicitly work out closed form solutions for the optimal hedging strategies (in the sense of Bouchaud and Sornette) in the case of European call options, where the underlying is modeled by (unbiased) iid additive returns with Student-t distributions. The results may serve as illustrative examples for option pricing in the presence of fat tails.
\end{abstract}




\section{Introduction}

Black and Scholes option pricing theory relies on the existence of a dynamic trading strategy in the underlying asset that exactly replicates the option holder's claim [1-4]. There is no risk for the writer, and the fair option price can be interpreted as the cost of the perfect hedging strategy.

Real world markets are, however, not complete, i.e., there are no riskless hedging strategies that would provide unique option prices. The incompleteness stems from a number of reasons. E.g., stock market returns are not log-normally distributed (as assumed in the Black Scholes analysis). It is widely agreed that marginal return distributions have fat tails, i.e., large events occur with significantly higher frequency than to be expected from the log-normal assumption.

An interesting approach to option theory for incomplete markets has been worked out by Müller [5], Föllmer and Sondermann [6], Schäl [7] and Schweizer [8], and also by Bouchaud and coworkers [9, 10], see also [11-15]. At the heart of these approaches lies a minimization of the risk inherent in writing the option. Different risk measures have been suggested, including the global and local variance of the cost process [5-7] and the variance of the global operator wealth 9].

In this article, I will explicitly work out closed form solutions for the optimal hedging strategies (in the sense of Bouchaud and Sornette) in the case of European call options, where the underlying is modeled by (unbiased) iid additive returns with Student-t distributions. The results may serve as illustrative examples for option pricing in the presence of fat tails. Furthermore, since Student distributions have been considered as acceptable models of market returns [16-20], the results presented here could also be useful for practical purposes.

This article is organized as follows: In section 2, I setup the model and notation. A number of useful formulae is derived that are of significant help in the explicit calculations of later sections. In section 3 we reproduce a few (well known) results for Gaussian returns. In section 4 I derive the closed form solutions $\rrbracket$ for the Student-t case. Illustrative example results are given in section 5. Conclusions follow. Some technical parts are deferred to three appendices.

\footnotetext{
${ }^{1}$ Closed form here means that the result is presented as a finite sum of terms built from standard functions.
} 


\section{Model Setup and Basic Formulae}

Consider a European call option, with maturity at $T=N \tau$, and strike price $x_{s}$. Trading and hedging is only possible at discrete times $t=k \tau$, $k=0, \ldots, N$. The underlying price process is given by $x_{k} \equiv x(k \tau)$. At expiry the option holder's claim is given by

$$
f\left(x_{N}\right)=\max \left(x_{N}-x_{s}, 0\right) .
$$

For the sake of simplicity we assume that the (risk free) interest rate is zero.

After writing an option, the bank will perform the following (hedging) actions. $k=0, \ldots, N-1$ : Trade the underlying such that the bank's portfolio contains $\phi_{k}$ pieces of stock. $k=N$ : Sell the portfolio and satisfy the option holder's claim.

Following Bouchaud and Sornette [9], we write down the global cost balance for the bank (per option issued): $2^{2}$

$$
C=\max \left(x_{N}-x_{s}, 0\right)-\sum_{k=0}^{N-1} \phi_{k}\left(x_{k}\right)\left(x_{k+1}-x_{k}\right) .
$$

If it were predictable (deterministic), $C$ would be the correct price of the option (ignoring transaction action costs etc.). However, as a function of the underlying, $C$ is a stochastic variable. The fluctuations of $C$ will in general depend on the choice of the hedging strategy $\phi$. In the Bouchaud Sornette approach, on chooses $\phi$ in order to make $C$ as determininistic as possible, e.g., by demanding that the variance of $C$ is minimal. The optimal hedging strategy $\phi^{*}$ is then determined by

$$
R^{2}\left(\phi^{*}\right)=\left\langle C^{2}\right\rangle-\langle C\rangle^{2}=\text { minimal }
$$

where $\langle$.$\rangle denotes averaging over the price process. For sufficiently small$ residual risk, e.g., for $R /\langle C\rangle \ll 1$, it is reasonable for the bank to define the option price as

$$
\text { price }=\langle C\rangle+\text { premium for the residual risk } .
$$

The extra premium for the residual risk will of course depend on the bank's risk preferences.

\footnotetext{
${ }^{2}$ We have not included the option premium in the balance.
} 
Let us now assume the following (simplified) model for the returns of the underlying asset:

$$
x_{k+1}-x_{k}=r_{k+1}, \quad k=0, \ldots, N-1,
$$

where the $r$ 's are iid with probability density function (pdf) $p(r)$. We require that $p$ should have a finite variance, defined by ${ }^{3}$

$$
\sigma^{2}=\int d r p(r) r^{2}
$$

However, we are not assuming that $p$ is Gaussian or log-normal. The stock price $x_{k}$ can be written as

$$
x_{k}=x_{0}+\sum_{i=1}^{k} r_{k} \equiv x_{0}+R_{k} .
$$

The probability measure is defined by

$$
\langle F\rangle=\int d r_{1} p\left(r_{1}\right) \ldots \int d r_{N} p\left(r_{N}\right) F\left(r_{1}, \ldots, r_{N}\right) .
$$

We will assume that $p(r)$ is even, i.e., $p(r)=p(-r)$. Then the expectation value of $C$ is unaffected by the hedging strategy $\phi:\langle C\rangle=\langle f\rangle$. In the present context it is given by

$$
\langle C\rangle=\int_{-\infty}^{\infty} d t P_{N}(t) \max \left(x_{0}+t-x_{s}, 0\right)=\int_{-\zeta}^{\infty} d t P_{N}(t)(\zeta+t)
$$

We have defined

$$
\zeta=x_{0}-x s .
$$

At $k=0$ the option is in-the-money, at-the-money, or out-of-the-money, for $\zeta>0, \zeta=0$, or $\zeta<0$, respectively.

$P_{l}$ is the pdf of $R_{l}$, i.e., the $l$-fold convolution of $p$. It can be conveniently accessed via Fourier transformation methods. Introducing the characteristic function $\tilde{p}(q)$ through

$$
\tilde{p}(q)=\int d r e^{i q r} p(r)
$$

we have

$$
\tilde{P}_{l}(q)=\tilde{p}(q)^{l} .
$$

\footnotetext{
${ }^{3}$ Integrals without explicit limits should always be extended over the whole real axis.
} 
It is not difficult to show that the hedging strategy minimizing the variance of $C$ can be expressed as

$$
\phi_{k}^{*}\left(x_{k}\right)=\frac{1}{\sigma^{2}} \int d r p(r) r \int d t P_{N-k-1}(t) f\left(x_{k}+r+t\right) .
$$

It is demonstrated in Appendix A that

$$
\psi_{k}^{*}=-\frac{1}{(N-k) \sigma^{2}} \int \frac{d q}{2 \pi} \frac{\sin (q \xi)}{q} \frac{1}{q} \frac{d}{d q} \tilde{p}(q)^{N-k}
$$

where $\phi_{k}^{*}=\frac{1}{2}+\psi_{k}^{*}$, and $\xi \equiv x_{k}-x_{s}$.

The residual risk can be expressed as

$$
R^{2}\left(\phi^{*}\right)=\left\langle f^{2}\right\rangle-\langle f\rangle^{2}-\sigma^{2} \sum_{k=0}^{N-1} \int d x P_{k}\left(x-x_{0}\right) \phi_{k}^{*}(x)^{2} .
$$

Here, $\left\langle f^{2}\right\rangle-\langle f\rangle^{2}$ is the unhedged risk. $\langle f\rangle$ and $\left\langle f^{2}\right\rangle$ can be computed with the help of the following relations, derived in Appendix B:

$$
\begin{aligned}
& \langle f\rangle=\zeta\left(\frac{1}{2}+L_{1}(\zeta)\right)+L_{2}(\zeta), \\
& \left\langle f^{2}\right\rangle=\zeta^{2}\left(\frac{1}{2}+L_{1}(\zeta)\right)+\zeta L_{2}(\zeta)+\left(\frac{1}{2} N \sigma^{2}+L_{3}(\zeta)\right),
\end{aligned}
$$

where

$$
\begin{aligned}
& L_{1}(\zeta)=\int \frac{d q}{2 \pi} \frac{\sin (\zeta q)}{q} \tilde{p}(q)^{N}, \\
& L_{2}(\zeta)=-\int \frac{d q}{2 \pi} \cos (\zeta q) \frac{1}{q} \frac{d}{d q} \tilde{p}(q)^{N}, \\
& L_{3}(\zeta)=-\int \frac{d q}{2 \pi} \frac{\sin (\zeta q)}{q} \frac{1}{q} \frac{d}{d q} \tilde{p}(q)^{N} .
\end{aligned}
$$

The second term in eq. (4) can be rewritten as

$$
\sigma^{2} \sum_{k=0}^{N-1} \int d \xi P_{k}(\xi-\zeta)\left(\frac{1}{2}+\psi_{k}^{*}(\xi)\right)^{2}
$$

In the examples to be discussed below we shall evaluate this term by performing the $\xi$-integration numerically, starting from closed form expressions for $P_{k}$ and $\psi_{k}^{*}$. 


\section{The Gaussian Case}

As a first application of the formulae derived in the previous section, and for later comparison with the Student-t case, let us consider the case of Gaussian returns, i.e., $p(r)=1 / \sqrt{2 \pi \sigma^{2}} e^{-r^{2} /\left(2 \sigma^{2}\right)}$. The characteristic function is $\tilde{p}(q)=e^{-\frac{1}{2} \sigma^{2} q^{2}}$. The integrals for $\psi_{k}^{*}$ and $L_{i}$ are elementary. With $\sigma_{l}^{2}=l \sigma^{2}$ we find

$$
\psi_{k, G}^{*}=\frac{1}{2} \operatorname{erf}\left(\xi / \sqrt{2 \sigma_{N-k}^{2}}\right) .
$$

For $\phi_{k, G}^{*}=\frac{1}{2}+\psi_{k, G}^{*}$ one thus obtains $\phi_{k, G}^{*}=\hat{N}\left(\xi / \sigma_{N-k}\right)$, where $\hat{N}$ denotes the cumulative distribution function of the normal distribution. For the $L_{i}$ we get

$$
\begin{aligned}
& L_{1, G}=\frac{1}{2} \operatorname{erf}\left(\zeta / \sqrt{2 \sigma_{N}^{2}}\right), \\
& L_{2, G}=\sigma_{N}^{2} \frac{e^{-\zeta^{2} /\left(2 \sigma_{N}^{2}\right)}}{\sqrt{2 \pi \sigma_{N}^{2}}}, \\
& L_{3, G}=\frac{1}{2} \sigma_{N}^{2} \operatorname{erf}\left(\zeta / \sqrt{2 \sigma_{N}^{2}}\right) .
\end{aligned}
$$

For a complete discussion of the Gaussian case see [9, 10].

\section{Options with Student-t Underlying}

We shall now consider the case that the return distribution is of the Student-t type,

$$
p^{(\mu)}(r)=\frac{\Gamma\left(\frac{1}{2}(1+\mu)\right)}{\sqrt{\pi} \Gamma\left(\frac{1}{2} \mu\right)} \frac{a^{\mu}}{\left(a^{2}+r^{2}\right)^{(1+\mu) / 2}} .
$$

$a>0$ is a scale parameter that we shall later use to fix the standard deviation. The latter exists for $\mu>2$. For $\mu$ an odd integer, one can derive relatively simple expressions for the characteristic functions $\tilde{p}^{(\mu)}$, thus making feasible closed form solutions for the hedge functions $\phi_{k}^{*}$. Let us therefore concentrate on the case $\mu=2 m+1$, with $m=1,2, \ldots$ By an elementary integration one can show that

$$
\sigma^{2}=\frac{a^{2}}{2 m-1}
$$


A straightforward but somewhat lengthy calculation (employing the theorem of residues) yields

$$
\tilde{p}^{(2 m+1)}(q)=e^{-a|q|} T^{[m]}(a|q|),
$$

where

$$
T^{[m]}(q)=\sum_{k=0}^{m}\left(\begin{array}{c}
m \\
k
\end{array}\right) /\left(\begin{array}{c}
2 m \\
k
\end{array}\right) \frac{(2 q)^{k}}{k !}
$$

is a polynomial of degree $m$. Here are a few examples:

$$
\begin{aligned}
& T^{[0]}(q)=1, \\
& T^{[1]}(q)=1+q, \\
& T^{[2]}(q)=1+q+\frac{1}{3} q^{2} .
\end{aligned}
$$

In Appendix C eq. (3) is used to derive a closed form expression for $\psi_{k}^{[m] *}$. The result is

$$
\psi_{k}^{[m] *}=\frac{1}{\pi} \arctan \frac{\xi^{\prime}}{N-k}+\sum_{l=1}^{m(N-k)-1} \frac{A_{l}^{[m, N-k]}(l-1) !}{\pi} \frac{\sin \left(l \arctan \left(\frac{\xi^{\prime}}{N-k}\right)\right)}{\left(\xi^{\prime 2}+(N-k)^{2}\right)^{l / 2}} .
$$

Here we have defined

$$
\xi^{\prime}=\frac{\xi}{a}=\frac{x_{k}-x_{s}}{a} .
$$

The coefficients $A_{j}^{[m, N-k]}$ are defined by

$$
T^{[m]}(q)^{N-k-1} T^{[m-1]}(q) \equiv \sum_{j=0}^{m(N-k)-1} A_{j}^{[m, N-k]} q^{j}
$$

Calculations very similar to those presented in Appendix $\mathrm{C}$ help us to find explicit expressions for the $L_{i}$, defined in eq. (6).

For $L_{1}$ we get

$$
L_{1}^{[m]}=\int_{0}^{\infty} \frac{d q}{\pi} \frac{\sin \left(\zeta^{\prime} q\right)}{q} e^{-N q} T^{[m]}(q)^{N},
$$

with $\zeta^{\prime}=\zeta / a$. Expanding

$$
T^{[m]}(q)^{N}=\sum_{l=0}^{m N} B_{l}^{[m, N]} q^{l}
$$


we find

$$
L_{1}^{[m]}=\frac{1}{\pi} \arctan \frac{\zeta^{\prime}}{N}+\sum_{l=1}^{m N} \frac{B_{l}^{[m, N]}(l-1) !}{\pi} \frac{\sin \left(l \arctan \left(\frac{\zeta^{\prime}}{N}\right)\right)}{\left(\zeta^{\prime 2}+N^{2}\right)^{l / 2}} .
$$

Similarly, for $L_{2}$ one obtains

$$
L_{2}=\frac{N \sigma^{2}}{a} \int_{0}^{\infty} \frac{d q}{\pi} \cos \left(\zeta^{\prime} q\right) e^{-N q} T^{[m]}(q)^{N-1} T^{[m-1]}(q) .
$$

Using that

$$
\int_{0}^{\infty} d x x^{\alpha-1} e^{-\beta x} \cos (\gamma x)=\frac{\Gamma(\alpha)}{\left(\beta^{2}+\gamma^{2}\right)^{\alpha / 2}} \cos \left(\alpha \arctan \frac{\gamma}{\beta}\right),
$$

one arrives at

$$
L_{2}^{[m]}=\frac{N \sigma^{2}}{a} \sum_{l=0}^{m N-1} \frac{A_{l}^{[m, N]} l !}{\pi} \frac{\cos \left((l+1) \arctan \left(\frac{\zeta^{\prime}}{N}\right)\right)}{\left(\zeta^{\prime 2}+N^{2}\right)^{(l+1) / 2}} .
$$

The calculation of $L_{3}$ goes along the same lines. We find

$$
L_{3}^{[m]}=N \sigma^{2}\left(\frac{1}{\pi} \arctan \frac{\zeta^{\prime}}{N}+\sum_{l=1}^{m N-1} \frac{A_{l}^{[m, N]}(l-1) !}{\pi} \frac{\sin \left(l \arctan \left(\frac{\zeta^{\prime}}{N}\right)\right)}{\left(\zeta^{\prime 2}+N^{2}\right)^{l / 2}}\right) .
$$

For the calculation of the residual risk we shall also need an expression for $P_{k}$, the $k$-fold convolution of $p$. The result is

$$
a P_{k}(a t)=\sum_{l=0}^{m k} \frac{B_{l}^{[m, k]} l !}{\pi} \frac{\cos \left((l+1) \arctan \left(\frac{t}{k}\right)\right)}{\left(t^{2}+k^{2}\right)^{(l+1) / 2}}
$$

\section{Example Results}

In this section we will present some example results for the Student-t distribution, in comparison with the Gaussian case. Let us start with a look at the hedge functions for different values of $m$. We choose $N=7$ and $k=0$. If you think of days as the hedging and trading intervals, this corresponds to one week before expiry. Figure 1 shows the results for $m=1,2$, and 3, together with the Gaussian results. $\psi_{0}^{*}$ is plotted as function of $\zeta / \sigma$, where $\sigma$ denotes the standard deviation (per day). 


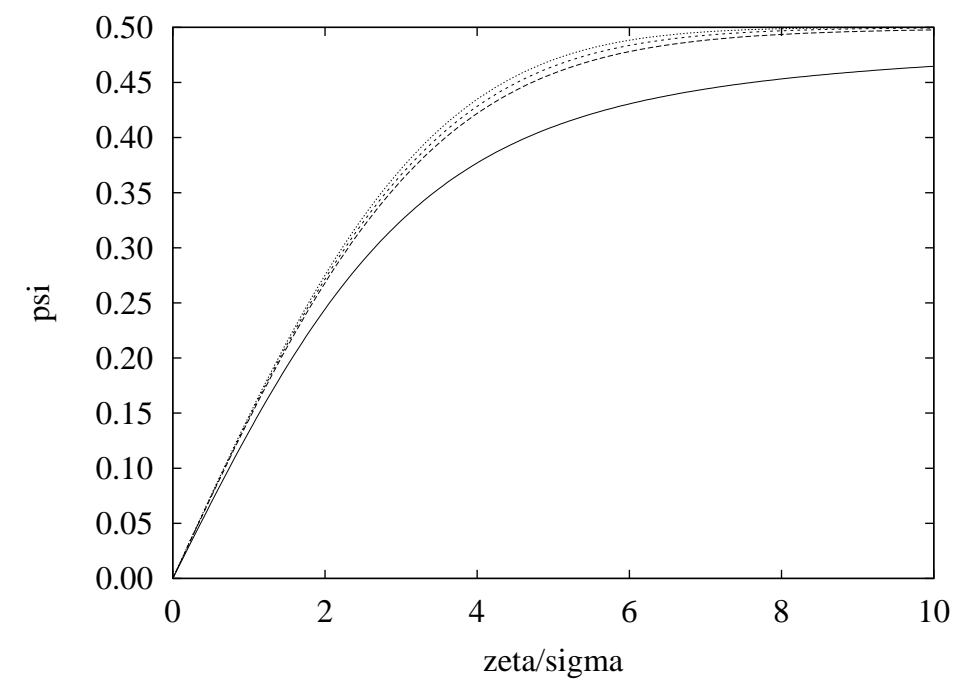

Figure 1: The $N=7, k=0$ hedging function $\psi_{0}^{*}$ for Student-t underlying with $m=1,2$, and 3 (bottom to top). The uppermost curve is the hedge function for the Gaussian case.

With increasing $m$, the curves converge quickly towards the $\psi$-function of the Gaussian returns. Closer to expiry the difference to the Gaussian behaviour is more significant, as demonstrated in Figure 2, which shows $\psi_{0}^{*}$ for the same set of $m$-values, however with $N-k=1$, i.e., one day before maturity.

Let us next consider the behaviour of $\langle C\rangle$. Figure 3 shows this quantity for $N=7$, for $m=3$, and for the Gaussian, as a function of $\zeta / \sigma$. Around $\zeta=0$, the Student result is a little bit below the Gaussian, farther out it is a very little bit above it ("volatility smile").

Figure 1 shows again results for $N=7,\langle C\rangle$ as a function of $\zeta / \sigma$, together with the risk $\left\langle C^{2}\right\rangle-\langle C\rangle^{2}$, plotted with the help of error bars. The upper plot gives these quantities for the unhedged case, i.e., for $\phi=0$. For the lower plot the optimal hedging was performed. In both cases, the data with the smaller risk belong to the Gaussian, the other ones to the Student-t with $m=1$. Note that there remains quite some risk even after the optimal hedging. It is only for the Gaussian that this residual risk can be removed in the limit of continous hedging. 


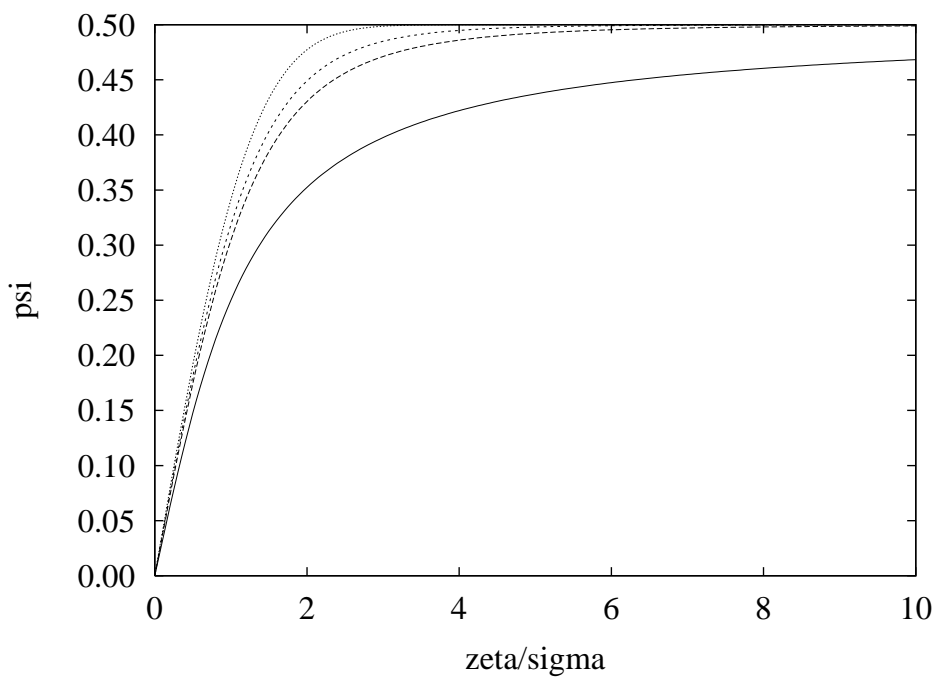

Figure 2: The $N=1, k=0$ hedging function $\psi_{0}^{*}$ for Student-t underlying with $m=1,2$, and 3 (bottom to top). The uppermost curve is the hedge function for the Gaussian case.

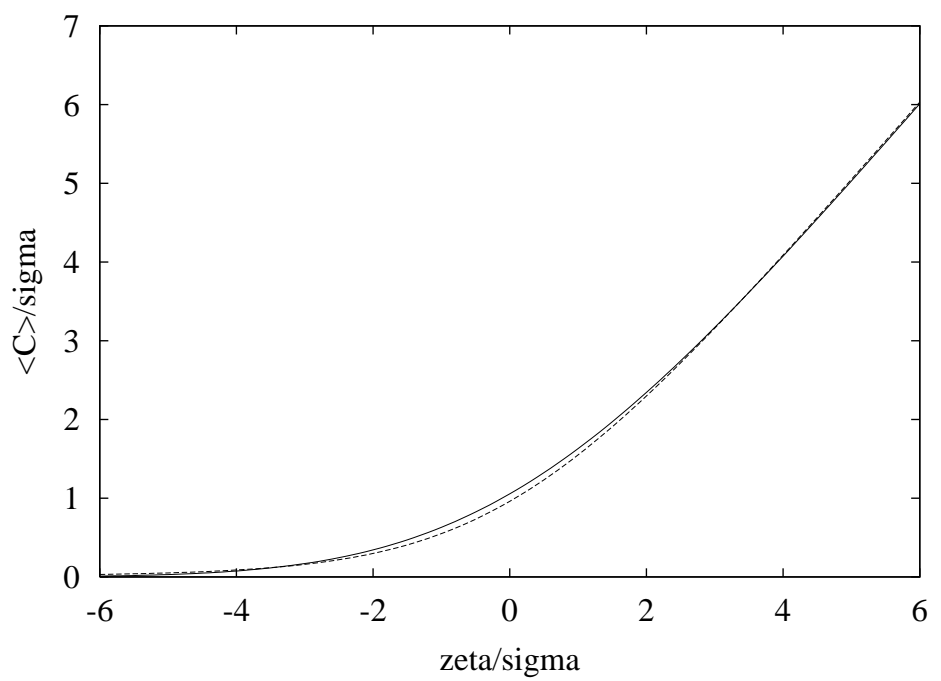

Figure 3: $\langle C\rangle$ for $N=7$, Student-t, with $m=3$, in comparison with the Gaussian. The Student-t result is below the Gaussian for small $\zeta$. 

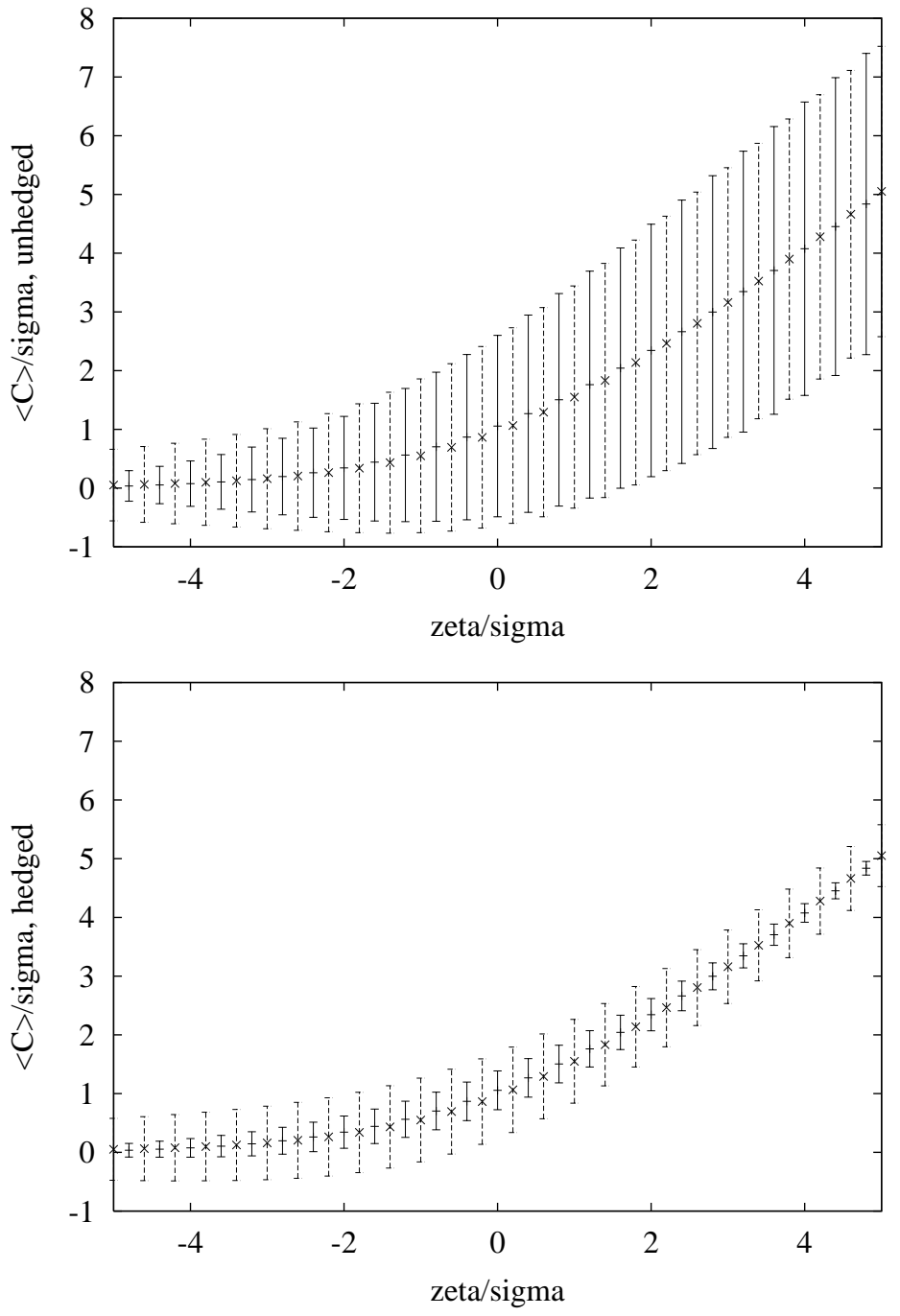

Figure 4: For $N=7$ : $\langle C\rangle$, together with $\left\langle C^{2}\right\rangle-\langle C\rangle^{2}$, plotted with the help of error bars. Top: unhedged, bottom: optimally hedged. Bars: Gaussian, crosses: Student-t with $m=1$. 


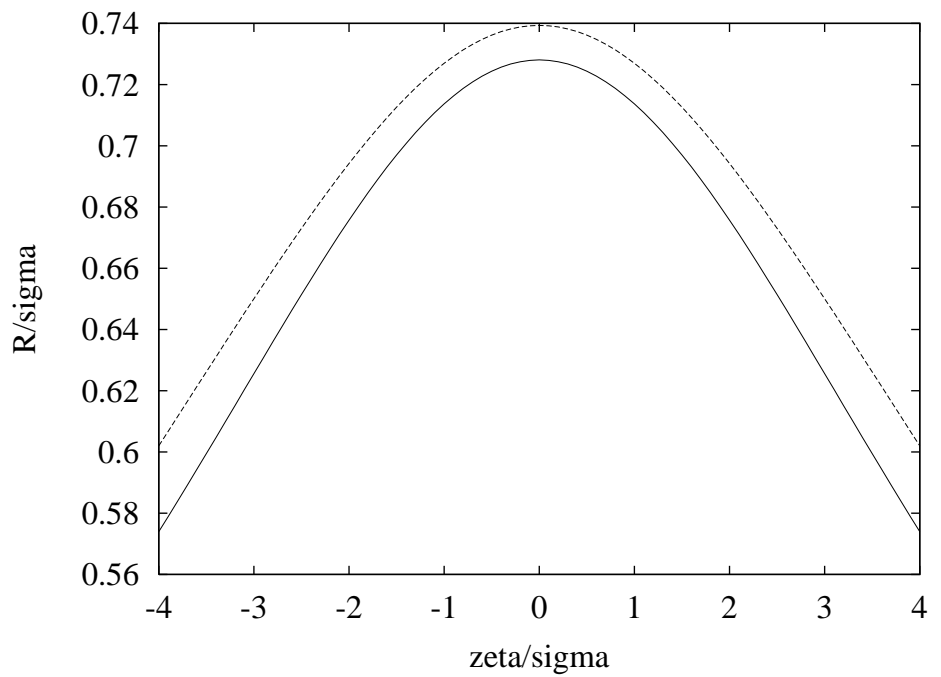

Figure 5: Upper curve: residual risk for $N=7$, Student-t with $m=1$, hedged with Gaussian hedge function. The lower curve is the residual risk in the case of optimal hedging.

It is interesting to visualize the "hedging error" occuring when one hedges the Student-t underlying with the Gaussian hedge functions given by eq. (77). The upper curve in Figure 5 denotes the residual risk for $N=7$, Student-t returns with $m=1$, hedged with the Gaussian hedge function (corresponding to the same standard deviation). The lower curve is the residual risk in the case of optimal hedging. The difference is not dramatic, but appreciable.

For non-Gaussian distributions, the variance is, of course, not always an appropriate measure of risk. It is therefore instructive to look at the full pdf of the quantity $C$. Given the hedge functions, it can be easily computed by Monte Carlo. In Figure 6 we see histograms (from 1 million samples of the whole hedging process) of $C(N=7)$ for the Gaussian and the $m=1$ Student-t returns, both optimally hedged. In the lower part of the figure (with $\log$ scale), we show additionally the function $1 /(C / \sigma)^{4}$, demonstrating that the power tail of the Student-t distribution is still around. For comparison: The amplitude in front of the $1 / x^{4}$ term in the 7 -fold convolution of the $m=1$ Student-t (with standard deviation one) is 0.24062 . If one assumes that the option writer would sell the option for $\langle C\rangle=0.96$, his value at risk 

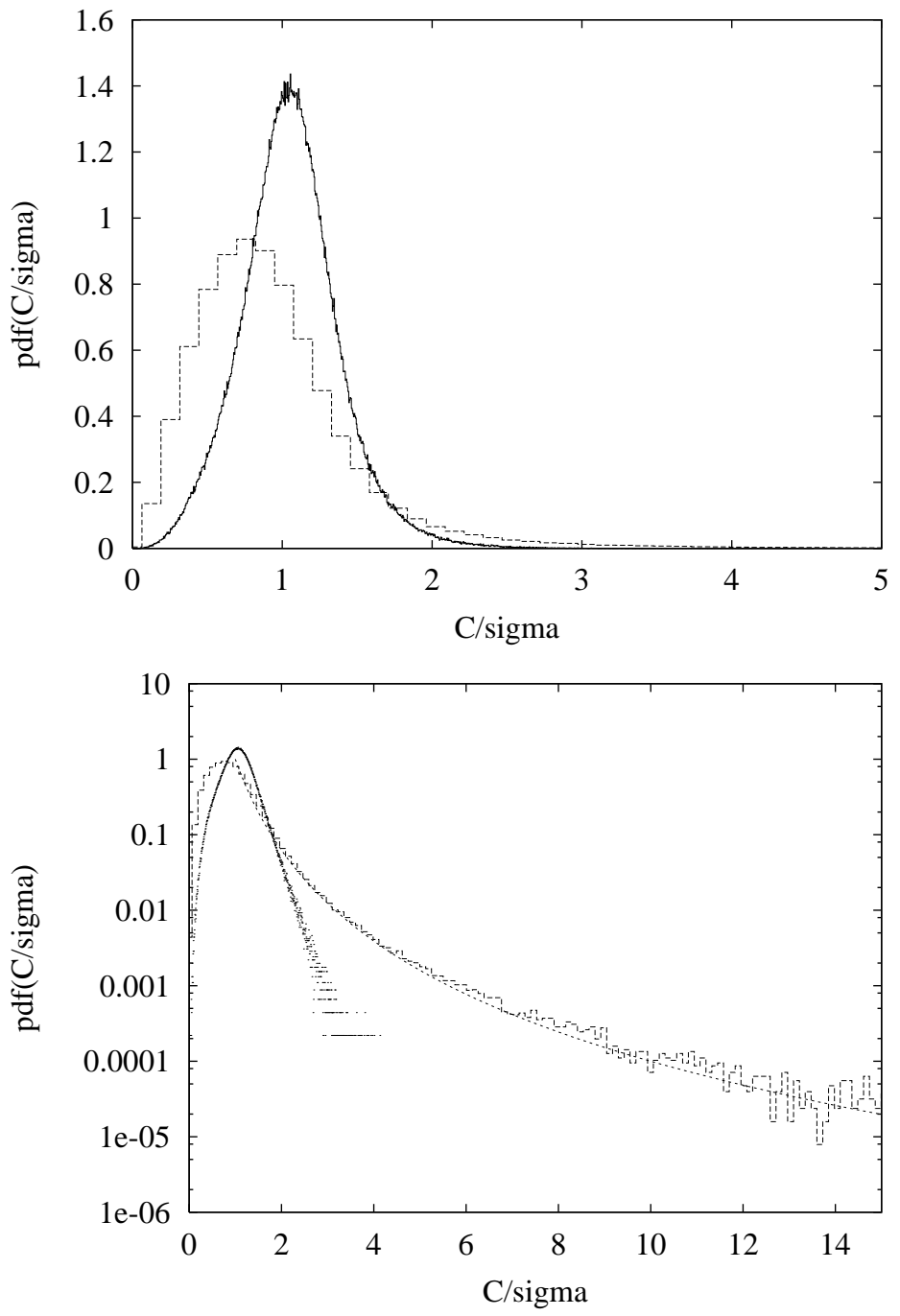

Figure 6: The pdf's of $C$, for $N=7$. Top: linear scales, bottom: vertical $\log$ scale. The distributions with the fatter tails belong to Student-t with $m=1$, the remaining stems from Gaussian returns. 
$(\operatorname{VaR}(5 \%))$ would be 0.98 , i.e., more than 100 percent of the option price!

\section{Conclusions}

In this paper, closed form solutions were derived for the optimal hedge functions in the case of unbiased Student-t returns. I hope that the results prove useful as illustrative examples for option pricing in the presence of fat tails, and as starting points for further investigations.

\section{Acknowledgement}

Discussions with J. Lemm and C. Wieczerkowski are gratefully acknowledged.

\section{References}

[1] R.C. Merton, Continuous Time Finance, Blackwell 1990

[2] J. Hull, Options, Futures and Other Derivative Securities, Prentice-Hall 1993

[3] D. Duffie, Dynamic Asset Pricing Theory, Princeton University Press 1996

[4] P. Wilmott, J. Dewynne, and S. Howison, Option Pricing, Oxford Financial Press 1997

[5] S. Müller, Arbitrage Pricing of Contingent Claims, Lect. Notes in Econ. and Math. Systems 254, Springer-Verlag, Berlin 1985

[6] H. Föllmer and D. Sondermann, Hedging of non-redundant contingent claims, in: W. Hildenbrand and A. Mas-Collel (eds.), Contributions to Mathematical Economics (1986) 205-223

[7] M. Schäl, On quadratic cost criteria for option hedging, Math. Oper. Res. 19 (1994) 121-131

[8] M. Schweizer, Variance-optimal hedging in discrete time, Math. Oper. Res. 20 (1995) 1-32 
[9] J.P. Bouchaud and D. Sornette, The Black-Scholes option pricing problem in mathematical finance: generalization and extensions for a large class of stochastic processes, J. Phys. I (France) 4 (1994) 863-881

[10] J.P. Bouchaud and M. Potters, Theory of Financial Risk, Aléa Saclay, Eyrolles, 1997.

[11] R. Frey, Derivative Asset Analysis in Models with Level-Dependent and Stochastic Volatility, working paper, Bonn 1997, available at http://addi.or.uni/bonn.de:1048/papers/bonnsfb401.htm

[12] E. Aurell and S. Simdyankin, Pricing risky options simply, Int. J. Theoretical and Applied Finance 1(1) (1998) 1-23

[13] O. Hammarlid, On minimizing risk in incomplete markets option pricing models, Int. J. Theoretical and Applied Finance 1(2) (1998) 227-233

[14] G. Wolczynska, An explicit formula for option pricing in discrete incomplete markets, Int. J. Theoretical and Applied Finance 1(2) (1998) $283-288$

[15] S. Fedotov and S. Mikhailov, Option Pricing Model for Incomplete Market, cond-mat/9807397

[16] T. Lux, The Stable Paretian Hypothesis and the Frequency of Large Returns: An Examination of Major German Stocks, Applied Financial Economics 6, (1996) 463

[17] M. Loretan and P. C. B. Phillips, Journal of Empirical Finance 1 (1994) 211

[18] P. Gopikrishnan, M. Meyer, L.A.N. Amaral, and H. E. Stanley, Inverse Cubic Law for the Probability Distribution of Stock Price Variations, European Journal of Physics B: Rapid Communications 3 (1998) 139140

[19] U. A. Müller, M. M. Dacorogna, and O. V. Pictet, in: A Practical Guide to Heavy Tails, R. J. Adler, R. E. Feldman and M. S. Taqqu (eds.), pp. 283, (Birkhäuser Publishers, 1998) 
[20] R. C. Blattberg and N. J. Gonedes, A Comparison of the Stable and Student Distributions as Statistical Model for Stock Prices, J. Business 47 (1974) 244-280

\section{Appendix A: Representations of $\phi_{k}^{*}$}

We start from

$$
\phi_{k}^{*}(x)=\frac{1}{\sigma^{2}} \int d r p(r) r \int d t P_{N-k-1}(t) f(x+r+t),
$$

with $f(x)=\max \left(x-x_{s}, 0\right)$. The claim function can be represented as follows:

$$
f(x)=-\int \frac{d q}{2 \pi} \frac{e^{i q\left(x-x_{s}\right)}}{(q-i \epsilon)^{2}} .
$$

To see this one evaluates the integral using the theory of residues.

$$
\int d q \frac{e^{i q \lambda}}{(q-i \epsilon)^{2}}=\left.2 \pi i \theta(\lambda) \frac{d}{d q} e^{i q \lambda}\right|_{q=i \epsilon}=-2 \pi \theta(\lambda) \lambda=-2 \pi \max (\lambda, 0) .
$$

Here, $\theta(\lambda)$ denotes the Heaviside step function. With $x_{k}-x_{s}=\xi$ we thus have

$$
\phi_{k}^{*}=-\frac{1}{\sigma^{2}} \int d r p(r) r \int d t P_{N-k-1}(t) \int \frac{d q}{2 \pi} \frac{e^{i q(\xi+r+t)}}{(q-i \epsilon)^{2}} .
$$

Exchanging the order of integration, we obtain

$$
\phi_{k}^{*}=-\frac{1}{\sigma^{2}} \int \frac{d q}{2 \pi} \frac{e^{i q \xi}}{(q-i \epsilon)^{2}} \int d r p(r) r e^{i q r} \int d t P_{N-k-1}(t) e^{i q t} .
$$

The $r$-integral can be written as $\frac{1}{i} \frac{d}{d q} \tilde{p}(q)$, whereas the $t$-integral is the same as $\tilde{p}(q)^{N-k-1}$. Putting things together we obtain

$$
\phi_{k}^{*}=-\frac{1}{(N-k) \sigma^{2}} \int \frac{d q}{2 \pi i} \frac{e^{i q \xi}}{(q-i \epsilon)^{2}} \frac{d}{d q} \tilde{p}(q)^{N-k} .
$$

To proceed further, we write $e^{i q \xi}=\cos (q \xi)+i \sin (q \xi)$. Then

$$
\phi_{k}^{*}=-\frac{1}{(N-k) \sigma^{2}} \int \frac{d q}{2 \pi i} \frac{\cos q \xi}{(q-i \epsilon)^{2}} \frac{d}{d q} \tilde{p}(q)^{N-k}+\psi_{k}^{*},
$$


with

$$
\psi_{k}^{*}=-\frac{1}{(N-k) \sigma^{2}} \int \frac{d q}{2 \pi} \frac{\sin (q \xi)}{q} \frac{1}{q} \frac{d}{d q} \tilde{p}(q)^{N-k} .
$$

In the expression for $\psi^{*}$ we have already performed the limit $\epsilon \rightarrow 0$. The first term of eq. (9) yields $1 / 2$. To see this we observe that the term is independent of $\xi$ : Differentiating with respect to $\xi$ makes the integrand an odd function of $q$ and the integral vanish. Without changing the integral we can therefore let $\xi=0$. Furthermore

$$
\begin{aligned}
- & \frac{1}{(N-k) \sigma^{2}} \int \frac{d q}{2 \pi i} \frac{1}{(q-i \epsilon)^{2}} \frac{d}{d q} \tilde{p}(q)^{N-k} \\
& =-\frac{1}{(N-k) \sigma^{2}} \int d x P_{N-k}(x) x \int \frac{d q}{2 \pi} \frac{1}{(q-i \epsilon)^{2}} \\
& =\frac{1}{(N-k) \sigma^{2}} \int_{0}^{\infty} d x P_{N-k}(x) x^{2} \\
& =\frac{1}{2} .
\end{aligned}
$$

This proves the relation $\phi_{k}^{*}=\frac{1}{2}+\psi_{k}^{*}$.

\section{Appendix B: Computation of $\left\langle f^{n}\right\rangle$}

The equation

$$
\left\langle f^{n}\right\rangle=\int d t P_{N}(t) \theta(\zeta+t)(\zeta+t)^{n}
$$

can be rewritten with the help of the following integral representation of the Heaviside step function:

$$
\theta(x)=\int \frac{d q}{2 \pi i} \frac{e^{i q x}}{q-i \epsilon},
$$

where $\epsilon>0$ is to be considered infinitesimal. We obtain

$$
\left\langle f^{n}\right\rangle=\int \frac{d q}{2 \pi i} \frac{1}{q-i \epsilon} \int d t P_{N}(t) e^{i q(\zeta+t)}(\zeta+t)^{n} .
$$

By elementary operations this can be rewritten as

$$
\left\langle f^{n}\right\rangle=\int \frac{d q}{2 \pi i} \frac{1}{q-i \epsilon}\left(\frac{1}{i} \frac{d}{d q}\right)^{n}\left(e^{i q \zeta} \tilde{p}(q)^{N}\right) .
$$


Working out the derivatives for $n=1,2$, we obtain the representation given in eqs. (5,6), provided we recognize the identities

$$
\begin{gathered}
\int \frac{d q}{2 \pi i} \frac{\cos (q \zeta)}{q-i \epsilon} \tilde{p}(q)^{N}=\frac{1}{2}, \\
-\int \frac{d q}{2 \pi i} \frac{\cos (q \zeta)}{q-i \epsilon} \frac{d^{2}}{d q^{2}} \tilde{p}(q)^{N}=\frac{1}{2} N \sigma^{2},
\end{gathered}
$$

and perform an integration by parts in one of the integrals. Eqs. (10,11) can be proved by first observing that both expressions do not depend on $\zeta$. Letting $\zeta=0$ thus leaves the integrals invariant. Transforming back to $t$-space we recognize integrals of $P_{N}(t)$ and $P_{N}(t) t^{2}$, respectively, for $t$ from 0 to $\infty$.

\section{Appendix C: Computing $\psi^{*}$ for Student-t}

We start from eq. (3) in the form

$$
\psi_{k}^{*}=\frac{1}{\sigma^{2}} \int_{0}^{\infty} \frac{d q}{\pi} \frac{\sin (q \xi)}{q} \tilde{p}(q)^{N-k-1}\left(-\frac{1}{q} \frac{d}{d q} \tilde{p}(q)\right) .
$$

It is easy to derive (by partial integration) the following recursion relation:

$$
\frac{1}{q} \frac{d}{d q} \tilde{p}^{(\mu)}(q)=-\frac{a^{2}}{\mu-2} \tilde{p}^{(\mu-2)}(q) .
$$

Employing this recurrence and eq. (8), we obtain

$$
\psi_{k}^{[m] *}=\int_{0}^{\infty} \frac{d q}{\pi} \frac{\sin (q \xi)}{q} e^{-(N-k) a q} T^{[m]}(a q)^{N-k-1} T^{[m-1]}(a q) .
$$

Performing the substitution $q \rightarrow q / a$ and defining

$$
\xi^{\prime}=\frac{\xi}{a}=\frac{x_{k}-x_{s}}{a}
$$

one obtains

$$
\psi_{k}^{[m] *}=\int_{0}^{\infty} \frac{d q}{\pi} \frac{\sin \left(q \xi^{\prime}\right)}{q} e^{-(N-k) q} T^{[m]}(q)^{N-k-1} T^{[m-1]}(q) .
$$


The product of the two $T$-factors is a polynomial of degree $m(N-k)-1$ :

$$
T^{[m]}(q)^{N-k-1} T^{[m-1]}(q) \equiv \sum_{l=0}^{m(N-k)-1} A_{l}^{[m, N-k]} q^{l} .
$$

E.g., for $m=1$, the $A$-coefficients are given by

$$
A_{l}^{[1, N-k]}=\left(\begin{array}{c}
N-k-1 \\
l
\end{array}\right) .
$$

For larger $m$ the expressions become more involved. They can however easily be obtained with the help of a computer algebra program.

Eq. (12) becomes

$$
\psi_{k}^{[m] *}=\frac{1}{\pi} \sum_{l=0}^{m(N-k)-1} A_{l}^{[m, N-k]} \int_{0}^{\infty} d q \frac{\sin \left(q \xi^{\prime}\right)}{q} e^{-(N-k) q} q^{l}
$$

This integral can be solved in closed form (Gradstein/Ryshik, 3.944):

$$
I(\alpha, \beta, \gamma)=\int_{0}^{\infty} d x x^{\alpha-1} e^{-\beta x} \sin (\gamma x)=\frac{\Gamma(\alpha)}{\left(\beta^{2}+\gamma^{2}\right)^{\alpha / 2}} \sin \left(\alpha \arctan \frac{\gamma}{\beta}\right) .
$$

We have to identify $\alpha=l, \beta=N-k$, and $\gamma=\xi^{\prime}$. Observing that

$$
\lim _{\alpha \rightarrow 0} I(\alpha, \beta, \gamma)=\arctan \frac{\gamma}{\beta} \text {, }
$$

we finally arrive at

$$
\psi_{k}^{[m] *}=\frac{1}{\pi} \arctan \frac{\xi^{\prime}}{N-k}+\sum_{l=1}^{m(N-k)-1} \frac{A_{l}^{[m, N-k]}(l-1) !}{\pi} \frac{\sin \left(l \arctan \left(\frac{\xi^{\prime}}{N-k}\right)\right)}{\left(\xi^{\prime 2}+(N-k)^{2}\right)^{l / 2}} .
$$

Note that this can alternatively be represented as

$$
\psi_{k}^{[m] *}=\frac{1}{\pi} \arctan \frac{\xi^{\prime}}{N-k}+\sum_{l=1}^{m(N-k)-1} \frac{A_{l}^{[m, N-k]}(l-1) !}{\pi} \frac{\operatorname{Im}\left(N-k+i \xi^{\prime}\right)^{l}}{\left(\xi^{\prime 2}+(N-k)^{2}\right)^{l}} .
$$

One might wish to expand the imaginary part

$$
\operatorname{Im}(a+i b)^{l}=\sum_{j=0}^{[(l-1) / 2]}\left(\begin{array}{l}
l \\
j
\end{array}\right)(-1)^{j} a^{l-2 j-1} b^{2 j+1},
$$

thus demonstrating that the corrections to the leading arctan behaviour decay like $\xi^{\prime-l}$ for large $\xi^{\prime}$. $[j]$ denotes the largest integer smaller or equal to $j$. 